\title{
Impact of longitudinal tumor location on postoperative outcomes in patients undergoing resection for gallbladder cancer: Fundus and body vs. neck and cystic duct
}

\author{
Sanghyun SONG, Kil Hwan KIM, Sungho JO*
}

Department of Surgery, Dankook University College of Medicine, Cheonan, Korea

Introduction: It is known that gallbladder cancer (GBC) in the neck or cystic duct (NC-GBC) has a better prognosis than GBC in the fundus or body (FB-GBC), but systematic studies on this are insufficient. We performed this study to investigate the impact of longitudinal tumor location on postoperative outcomes in patients undergoing resection for GBC.

Methods: A retrospective study was conducted for patients who underwent a radical resection for GBC from February 2008 to November 2017 at the Dankook University Hospital. A total of 98 patients underwent surgery for GBC, of which 77 patients who underwent curative intent surgery were included in the study. They were classified into FB-GBC and NC-GBC groups according to longitudinal tumor location, and the postoperative outcomes were compared and analyzed.

Results: There were no significant differences in the clinicopathological characteristics, TNM stage, postoperative complications, and in-hospital mortality between two groups. However, NC-GBC significantly showed more sclerotic gross type, poorer differentiation, and more lymphatic and perineural microinvasion. The radical resection rate was statistically higher in FB-GBC group (93.1\% vs. $73.7 \% ; p=0.036$ ) and adjuvant 5-FU based CCRT was more carried out in NC-GBC group (19.0\% vs. 57.9\%; $p<0.001)$. The recurrence rates after surgery was statistically higher in NC-GBC group $(25.9 \%$ vs. $52.6 \%, p=0.047)$, but there were no differences in disease-free survival (DFS) and overall survival (OS).

Conclusions: Although NC-GBC showed more aggressive microscopic pathological findings and higher recurrence rate than FBGBC, there were no differences in DFS and OS according to longitudinal tumor location of GBC. 